Cinémas

Revue d'études cinématographiques

Journal of Film Studies

\title{
Are you talking to me? Les enjeux du women's cinema pour un regard féministe
}

\section{Chantal Nadeau}

Volume 2, numéro 2-3, printemps 1992

Cinéma et Réception

URI : https://id.erudit.org/iderudit/1001083ar

DOI : https://doi.org/10.7202/1001083ar

Aller au sommaire du numéro

Éditeur(s)

Cinémas

ISSN

1181-6945 (imprimé)

1705-6500 (numérique)

Découvrir la revue

Citer cet article

Nadeau, C. (1992). Are you talking to me? Les enjeux du women's cinema pour un regard féministe. Cinémas, 2(2-3), 171-191. https://doi.org/10.7202/1001083ar
Résumé de l'article

À partir d'une analyse critique et féministe de la construction d'un regard sexué au cinéma, le présent texte confronte deux pratiques

cinématographiques divergentes : le women's cinema et le woman's film. L'argumentation vise principalement à voir comment chacune des pratiques cinématographiques articule une représentation de la femme selon l'axe objet/sujet du regard. Trois analyses filmiques sont ainsi menées de front : The Berlin Affair de Liliana Cavani et A Winter Tan de Jackie Burroughs, reconnues comme des stratégies de référence, répondant au projet politique du women's cinema et Thelma \& Louise de Ridley Scott positionné sous l'angle remodelé mais traditionnel du woman's film. 


\title{
Are you talking to me? \\ Les enjeux du women's cinema pour un regard féministe
}

\section{Chantal Nadeau}

\section{RÉSUMÉ}

\begin{abstract}
À partir d'une analyse critique et féministe de la construction d'un regard sexué au cinéma, le présent texte confronte deux pratiques cinématographiques divergentes: le women's cinema et le woman's film. L'argumentation vise principalement à voir comment chacune des pratiques cinématographiques articule une représentation de la femme selon l'axe objet/sujet du regard. Trois analyses filmiques sont ainsi menées de front: The Berlin Affair de Liliana Cavani et $A$ Winter Tan de Jackie Burroughs, reconnues comme des stratégies de référence, répondant au projet politique duwomen's cinema et Thelma \& Louise de Ridley Scott positionné sous l'angle remodelé mais traditionnel du woman's film.
\end{abstract}

\begin{abstract}
Through a critical feminist analysis of the construction of a gendered cinematic gaze, this article contrasts two differing film practices: "women's cinema" and the "woman's film.» The author argues that these cinematographic practices articulate a representation of women in terms of the object/suject axis of the gaze. Three filmanalyses are presented. The first two, The Berlin Affair by Liliana Cavani and $A$ Winter Tan by Jackie Burroughs provide reference strategies for the political project of "women's cinema», while Ridley Scott's
\end{abstract}


Thelma and Louise is seen as a remodelled but still traditional «woman's film.»

Le présent texte vise à questionner les articulations entre théories féministes et pratiques de femmes dans la constitution d'un regard féministe au cinéma. Plus spécifiquement, j'interrogerai la construction d'un regard féministe au cinéma par la confrontation de pratiques cinématographiques qui relèvent du women's cinema en regard du nihilisme qui caractérise la tradition du woman's film.

Il sera donc question, au cours de cet essai, de films, de pratiques cinématographiques qui subvertissent l'ordre d'une représentation qui prend pour acquis que le regard au cinéma a la même couleur que sa voix: blanc, hétérosexuel, mâle.

Il sera question, ici, de trois fables: une première qui parle d'une femme qui disparaît, une seconde qui parle d'une femme qui réapparaît, et une dernière qui parle d'une femme qui s'en va. Trois fables qui gravitent autour d'une seule et même histoire: celle du regard critique qui marque la représentation des femmes dans le women's cinema. Trois fables donc qui ont un point en commun: l'absence d'une morale. Ces trois fables toutefois ne sauraient exister sans l'épilogue de bon ton qui clôt la séquence. Un épilogue qui rappelle que le regard de la spectatrice au cinéma ne s'inscrit pas là où justement la tradition du woman's film voudrait l'y retrouver.

\section{Fable n 1: Une femme frappée de disparition}

«Regarder comment regarde celui qui regarde, et qui parfois me regarde...» (Le Doeuff, p. 91).

Il y a de cela quelque temps, au mois de novembre 1990 pour être plus précise, la Grande-Bretagne subissait un choc digne de la dérive des continents; Maggie - eh oui! - la Dame de fer se faisait ravir le pouvoir par des députés conservateurs terrifiés à l'idée de perdre les prochaines élections. À cette occasion, la revue New Statesman and Society publiait en première page de son édition du 16 novembre $1990^{1}$ une photo incroyable d'une Margaret Thatcher (voir photo) s'effaçant devant le regard péné- 
trant d'un Michael Heseltine, son principal adversaire à l'époque. Sans prétendre ici faire une sémiologie de l'image, j'aimerais néanmoins attirer l'attention sur deux références importantes pour une stratégie de regard auxquelles renvoit cette page couverture.

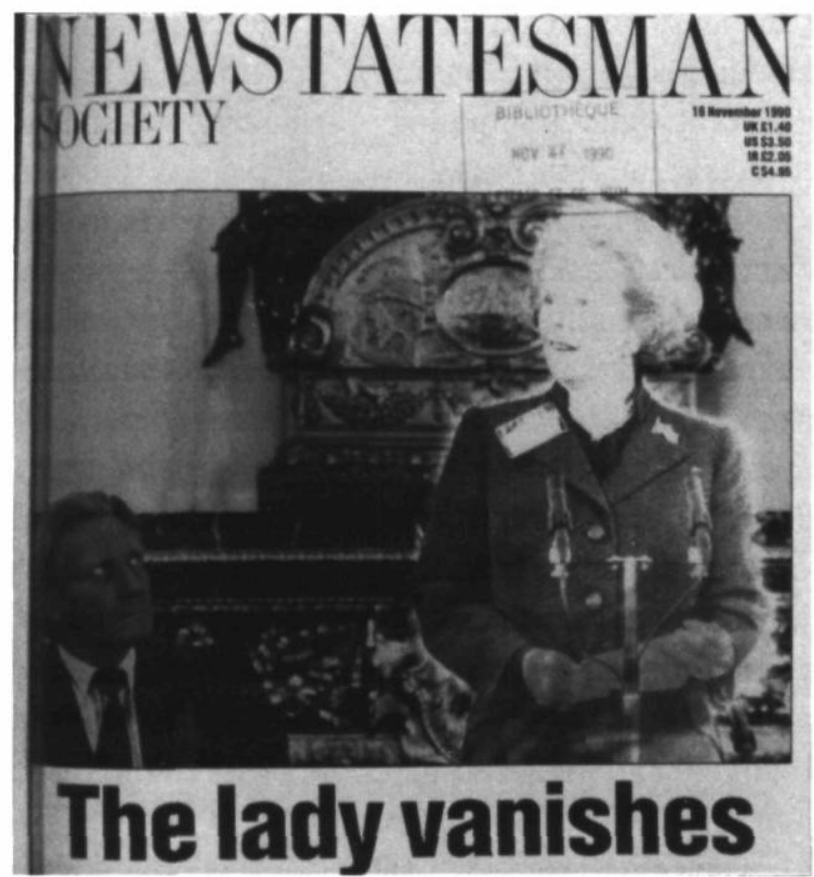

Premièrement, le titre lui-même. Une référence directe au film d'Alfred Hitchcock, The Lady Vanishes (G.-B., 1938). Un Hitchcock chez qui la femme, comme allait le démontrer le développement de la théorie féministe, tout en étant le point de convergence du regard, n'en est pas moins perpétuellement menacée d'être frappée de disparition. Deuxièmement, la photo ellemême. En voyant cette photo, je ne peux m'empêcher de penser à ces innombrables corps de femmes frappées de disparition dans l'histoire du cinéma, des femmes mises en transparence par des regards d'hommes. Car la photo a quelque chose d'hallucinant et de complètement pervers: celle de recouper dans un même espace l'intention de celui qui, jusqu'alors, n'était que spectateur 
(Michael Heseltine) et celle qui, statufiée (Margaret Thatcher), est transpercée par le regard qui l'efface ${ }^{2}$. Voilà, il me semble, une représentation corroborant tout à fait l'ordre de ce que serait le regard mâle au cinéma: une façon de faire disparaître ce qui est là, outrageusement réel, sous les yeux, pour mieux y transposer sa propre vision de l'autre, ici une femme frappée de disparition. Certains diront qu'il n'est question ici que de discours. À ceuxlà, je réponds que la représentation de l'image a force de mots: Maggie n'étant plus; les mauvaises langues ne manquant pas d'ajouter, Heseltine non plus.

L'analogie de cette page couverture avec le regard au cinéma est révélatrice des tensions qui existent entre la représentation de la femme au cinéma et la construction du regard. Le corps des femmes dans l'histoire du cinéma a toujours été mis en transparence. Et par mise en transparence, j'entends tout simplement le pouvoir qu'a le dispositif cinématographique de constituer une représentation de la femme qui, tout en étant là, s'efface au profit du véritable sujet du film: l'homme blanc, hétérosexuel.

Teresa de Lauretis utilise l'expression technology of gender ${ }^{3}$ pour parler du dispositif cinématographique du cinéma traditionnel, classique. L'expression évoque à la fois le raffinement et l'ordre derrière l'image cinématographique: le raffinement du traitement cinématographique classique, traditionnel et la nature de l'ordre du pouvoir (patriarcal) qui le constitue. Plus concrètement, dans l'histoire du cinéma, cela a signifié la cristallisation des rapports hommes-femmes selon l'axe objet-sujet: objet d'un regard (les femmes) / sujet du regard (les hommes).

Un des enjeux du women's cinema repose justement sur ce défi de faire de la représentation des femmes au cinéma non pas un objet de regard, mais bel et bien un sujet de regard ${ }^{4}$. Et, comme l'a souligné Teresa de Lauretis, la constitution d'un sujet femme pour le women's cinema passe nécessairement par la question d'une adresse à un regard de femme ${ }^{5}$. Dès lors, le processus de l'énonciation est intimement lié à la réception: une question comme «qui parle?» n'a de sens que si elle trouve prise dans celle du sujet à qui s'adresse le film.

Dès lors, se profile la question de celle qui regarde: la spectatrice. Et cette spectatrice, quoiqu'on en pense et quoiqu'en dise 
une certaine tendance effective dans la théorie cinématographique, a un «corps» - bien réel celui-là — et de surcroît que le cinéma suggère, un corps que le cinéma positionne dans son rapport à une représentation. Pour Meaghan Morris, la question d'un regard sexué au cinéma implique que la notion de spectatrice doit être comprise comme produit historique ${ }^{6}$. C'est donc dire que la question d'un regard féminin au cinéma renvoie non seulement à l'idée d'un sujet sexué, mais également aux références historiques et sociales qui construisent une certaine vision de la différence sexuelle dans la société. Et puisque la représentation, comme le défend Gayatri Spivak ${ }^{7}$, c'est à la fois parler de l'autre et parler pour l'autre, interroger la représentation au cinéma ne concerne pas seulement l'espace de l'écran, mais aussi le discours social qui fait vivre l'événement «cinéma». C'est en ce sens qu'une analyse féministe de la spectatrice au cinéma ne saurait faire l'économie des discours culturels dans lesquels la spectatrice se positionne en tant que sujet. On parle donc ici de rapports de pouvoir: de rapports de pouvoir entre les sexes. Et ce sont ces rapports de sexes qu'interpelle la représentation cinématographique de la femme dans le women's cinema.

Il importe de questionner le women's cinema non seulement dans sa définition conceptuelle, référant à un «genre» cinématographique, mais également comme stratégie de référence ${ }^{8}$ visant à introduire une autre façon de regarder et de représenter au cinéma. Une analyse du regard au cinéma, comme le suggère Meaghan Morris, doit pouvoir rendre compte du film «(...) as a complex event, rather than as a text in a viewing situation» (1989, p. 244). En somme, il s'agit de dépasser le moment du film pour mieux saisir la circulation des discours dans la société; pour mieux articuler des mécanismes contextuels qui structurent la différence de sexes, et ce tant à l'échelle des stratégies d'énonciation qu'au niveau du regard que porte la spectatrice sur le film ${ }^{9}$.

J'aimerais poursuivre ma réflexion en regardant de plus près quelques exemples de films qui jouent et déjouent certains repères traditionnels dans la façon de poser un regard sexué sur le film. Des films qui transgressent continuellement les frontières de reconnaissance pour la spectatrice. Des exemples qui, pour moi, maintiennent une conception de l'autre autrement, sans pour 
autant la frapper de disparition.

Parlons donc de ces films qui se veulent des tentatives de réinscrire la femme comme sujet. Il est de ces films qui, résolument, presque dans un a priori, sollicitent directement un regard critique de femme, lire ici un regard féministe. Il est de ces films qui, sans ambiguïté, appellent une adhésion à une politique de l'auteure: celle qui sait, qui tire sans fin à boulet rouge sur la marginalisation des femmes dans l'histoire. On pense tout de suite à Born in Flames (É.U.,1983) de Lizzie Borden ou encore Privilege (É.U.,1990) d'Yvonne Rainer.

Mais il y a aussi ce que je qualifierais de films du malaise. Des films non pas paniques, ni symptomatiques, mais des films provocants. Provocants dans leur délire, provocants dans leur marquage au fer rouge de la sexualité du statu quo, provocants en somme dans leur entêtement à refuser.

Ces films - The Berlin Affair (Interno Berlinese, 1986) de Liliana Cavani et $A$ Winter Tan (1987) de Jackie Burroughs —, je les ai choisis justement parce qu'ils ne sont pas transparents, parce qu'ils laissent en plan le regard tout en plaquant impitoyablement le corps dans une contrainte ambiguë à l'hétérosexualité ${ }^{10}$.

\section{Fable no 2: Une femme réapparaît - The Berlin Affair}

Les films de Liliana Cavani représentent, comme le souligne Giuliana Bruno, un véritable défi pour une analyse féministe du cinéma $^{11}$. D'une part, parce qu'ils ne s'inscrivent pas dans un discours féministe. D'autre part, parce que Cavani n'arrime pas son discours à une problématique de femmes à proprement dit, préférant donner aux hommes le privilège d'occuper l'avant-plan narratif et cinématographique. On a d'ailleurs souvent dit de Cavani qu'elle se pliait plus volontiers au plaisir d'explorer les fantasmes masculins que les fantasmes féminins.

Kaja Silverman est une des rares théoriciennes féministes à s'être intéressée à l'œuvre de Cavani. Pour Silverman, il est clair que les films de Cavani sont construits de telle sorte qu'ils forcent un rapport de séduction, voire une identification de la spectatrice avec la position qu'occupent les personnages masculins ${ }^{12}$. Toutefois, pour Silverman, cela ne veut pas dire pour autant le respect des conventions d'un regard mâle: au contraire, dit-elle, les films 
de Cavani font éclater un traitement traditionnel des rapports hommes-femmes dans un rapport hétérosexuel ${ }^{13}$. En effet, Cavani se plaît à subvertir le regard mâle dans ses films, sans pour autant appeler un regard féminin, par cette façon très particulière qu'elle a de soutirer le regard à une identification sous le mode de la concordance des sexes $^{14}$.

Peut-être est-il présomptueux de situer son cinéma du côté de la dénonciation politique. Toutefois, il est une chose dont les films de Cavani sont porteurs: c'est le refus d'absoudre la sexualité des rapports de pouvoir. En outre, par la reconduction perpétuelle dans ses films des liens qui se tissent entre le fascisme et les rapports intimes. C'est dans la virulence de ses images que Cavani inscrit peut-être le plus sa transgression face aux conventions d'un regard mâle. Qu'on pense à Portier de Nuit (1974), Au-delà du bien et du mal (1977), La Peau (1981), Derrière la porte (1983), tous rendent compte d'un profond malaise: celui de la fascination du pouvoir et du contrôle que peut exercer l'homme sur le corps et l'esprit de l'autre: la femme.

Avec The Berlin Affair, Cavani pousse la provocation plus à fond en substituant à la domination homme-femme, la domination d'une femme sur un couple marié. L'intrigue du film se trame sur l'éternel triangle amoureux. Par ailleurs la pierre angulaire, ici, ce n'est pas un des époux Von Hollendorf, mais l'amante elle-même, Mitsuko, objet de désir de la part des deux conjoints. La relation sera d'abord de nature lesbienne entre Louise, l'épouse d'un hautfonctionnaire sous le III ${ }^{e}$ Reich, et Mitsuko, la fille de l'ambassadeur japonais à Berlin. Par la suite, quand Heinz lui-même s'éprend de Mitsuko, la relation se poursuit à trois, mais dégagée du rapport de couple qui unit Louise et Heinz, condition formulée par Mitsuko qui revendique l'exclusivité du corps des deux époux.

Cavani rend très bien compte du pouvoir de fascination qu'exerce l'orientalisme sur le sujet occidental et le fantasme sexuel que représente l'exotisme de la japonaise. La représentation donnée de Mitsuko joue d'ailleurs continuellement sur l'ambiguïté de l'image traditionnelle de la geisha (exotisme, érotisme, fragilité) et sur la suspicion occidentale envers l'hermétisme oriental. Qui plus est, le film ne se contente pas de moduler les rapports lesbiens 
à une quelconque fascination pour l'étrangeté, mais fait état également de l'interdit social qui sanctionne l'homosexualité, notamment par un incessant rappel des purges qui frappent l'étatmajor nazi.

Mais ce qui est fascinant dans The Berlin Affair, c'est le plaisir que prend Cavani à déplacer l'ordre du regard, passant du point de vue de Louise qui raconte l'histoire, mais qui la vit également, à celui de Heinz, son mari, suscitant ainsi un renversement dans la position du regard chez la spectatrice. Si les deux tiers du film adoptent le point de vue de Louise dans son attirance pour cette «poupée de porcelaine ${ }^{15}$ » japonaise qu'est Mitsuko, le point de vue change brusquement et sans autre préambule lorsque Heinz s'éprend à son tour de Mitsuko.

The Berlin Affair est doublement intéressant pour une analyse féministe de la spectatrice dans le women's cinema. D'une part, parce qu'il amorce un changement dans la position de Cavani en donnant davantage prise à un regard féminin; d'autre part, parce qu'il respecte son principe moteur de défier l'identification sur la base d'une confrontation entre ce qui est dit, ce qui est représenté et ce qui regardé. Le film, tout en évoquant le pouvoir de manipulation de Mitsuko, ne joue jamais la carte de la dénonciation. Il suggère plutôt que c'est la méfiance et le pacte d'abstinence entre les deux époux qui constituent la base même de la relation entre les trois.

Ainsi, le cinéma de Cavani, loin d'appeller l'adhésion, la reconnaissance spectatorielle, précipite, au contraire, la fuite du regard devant la perversité des rapports de domination qui se tissent entre les protagonistes: une représentation qui renvoie à une implacable relation d'assujettissement des personnages aux rapports de domination dans la définition de la sexualité. Et si, justement, c'était cet assujettissement qui empêchait l'inscription du regard pour un sujet sexué? Si devant l'insoutenable s'opérait un acte de refus, proche de la répulsion - et ce refus, non seulement émotionnel, mais également rationnel? Et si, justement, ce qui choque chez Cavani, c'est l'impossibilité de s'identifier?

Peut-être qu'aux yeux d'un Raymond Bellour passerais-je pour une masochiste ${ }^{16}$, mais je comprends les films de Cavani, et plus particulièrement Berlin Affair, comme le lieu complexe où écla- 
tent les conventions du regard sexué au cinéma, un regard inexorablement reconduit par la logique des genres.

$\mathrm{Si}$, parfois, Cavani verse dans le trop-plein, son cinéma ne trahit jamais la moindre concession à une reconnaissance aveugle. Il n'y a pas de fantaisie, ni d'allégorie dans le cinéma de Cavani. Il n'y a que de la violence larvée qui éclate dans des étreintes mortelles. Sa représentation complexe et souvent ambiguë de la sexualité est à l'enseigne même du comment elle orchestre les manifestations du fascisme: dans ses lieux les plus intimes. Toutefois, Cavani ne lâche jamais de vue l'arrière-fond social d'une société bien-pensante dont les moindres gestes sont dictés par des règles douteuses: le respect des codes partisans. Avec The Berlin Affair, Cavani pousse jusqu'au paroxysme les rapports de pouvoir en exacerbant les tyrannies de l'intimité.

C'est en ce sens que l'œuvre de Cavani est transgressive pour une critique féministe du regard sexué traditionnel. Souvent décriée comme cinéaste maudite, et ce tant par les critiques masculins que féministes, Cavani incite à la répulsion: celle du choc que suscite le traitement délibérément interverti des rapports de domination. Et même un regard critique féministe y perd ses points de repères. Quand, par exemple, De Lauretis se demande si Cavani fait dans le woman's film ${ }^{17}$ plus qu'une simple distinction conceptuelle (entre woman's film et women's cinema), ce sont les codes de reconnaissance consacrés du cinéma de femme qu'elle interroge. Ceux susceptibles d'inscrire enfin la femme comme sujet de son propre regard, c'est-à-dire de sa propre expérience, répondant ainsi à une volonté de constituer une représentation de la femme qui réponde à la double convergence d'un cinéma fait par et pour les femmes ${ }^{18}$. Et la façon particulière priviliégiée par Cavani, c'est de lier, voire de confondre une critique politique de la sexualité par l'inscription d'une réification de l'érotisme chez les femmes.

Le cinéma de Cavani défie tellement les règles élémentaires d'un regard sexué (lire d'une division sexuelle qui répond aux rapports de pouvoir entre objet de regard et sujet de regard) que Sue Stewart ne peut s'empêcher de poser la question paradoxale, voire absurde mais qui m'apparaît quelque part légitime d'un point de vue féministe: «Is Liliana Cavani really a woman? ${ }^{19}$ » Et 
c'est là que réside l'intérêt du traitement de Liliana Cavani pour une articulation politique, historique et esthétique du regard de femme au cinéma: dans l'incessant questionnement sur la légitimité de la représentation des femmes au cinéma pour une position d'énonciation qui, à défaut d'être étiquetée féministe, n'en contribue pas moins à bouleverser l'ordre traditionnel. Car, comme le note pertinemment Stewart, et ce en parlant de Derrière la Porte, mais qui correspond tout autant aux autres films de Cavani comme Berlin Affair ou même Portier de Nuit, «(...) it seems that a conventional feminist critique using criteria of social protection, reverse sexism, and condemnation of negative behaviour simply does not work, because Cavani's films confound that perspective» (p. 37). Dès lors, poursuit-elle, pour un regard féministe, et l'insulte suprême est lancée, «(...) her ideas are suspect because they are revisionist. She has managed to defy the stereotype by having her female characters in Derrière la porte admit to her own desire for dominance; in this way she acknowledges that women, too, have a baser side and that they may even have an instinct for power» (p. 37). En somme, chez Cavani, le rapport à la sexualité pour les femmes explore les mêmes lieux que ceux des hommes, des lieux inexorablement marqués par des rapports de pouvoir non pas entre les sexes, mais entre les individus ${ }^{20}$. Dès lors, le regard de la spectatrice perd littéralement prise face à ces images où s'entrechoquent des univers où la perversion de la domination fait office de règle du jeu.

\section{Fable no 3: Une femme s'en va $-A$ Winter Tan}

Avec $A$ Winter Tan, la difficulté pour accrocher ou esquiver le regard de la spectatrice est d'un tout autre ordre. Plus que jamais, ici, l'affirmation de Teresa de Lauretis comme quoi la construction du genre peut être aussi tributaire de sa déconstruction trouve point d'appui. En effet, avec $A$ Winter Tan, ce n'est pas l'édification d'une représentation qui prime mais la déconstruction systémique d'un rapport critique au corps de la femme tel que défendu par 20 ans de théories et de luttes féministes.

Alors qu'avec Cavani, le regard sexué de la femme est sans cesse ramené à une dimension individuelle, celle de la femme non pas comme sujet social, mais la femme comme l'expression d'un 
désir au féminin, avec $A$ Winter Tan, l'inscription de la représentation du corps de la femme, si elle se veut une adresse directe à un regard féminin, n'en interpelle pas moins une cible collective bien précise: les spectatrices féministes. Et il ne s'agit pas ici de n'importe lesquelles féministes: il s'agit de féministes intellectuelles.

En le qualifiant de postféminisme, Elspeth Probyn résume très bien le contexte idéologique du film dans lequel s'inscrit $A$ Winter $\operatorname{Tan}^{21}$. Et le postféminisme tel que vécu par le personnage central Maryse consiste à reconstruire l'ordre d'un rapport à la sexualité/ nature bousculé par 20 ans ans de luttes féministes, opérant un juste retour historique: «There's feminism and then there's fucking.» L'heure n'est plus à la théorisation, pas plus d'ailleurs qu'à la distance critique aseptisée. Pour Maryse, le mot d'ordre sera désormais celui d'un travail d'antithèse pour ériger son propre corps comme la synthèse parfaite d'une Maryse postmoderne à savoir, comme elle le dit: «My natural sluttishness.»

Comme le souligne Probyn, en tant que féministes, c'est d'abord un profond malaise que l'on ressent face au discours vindicatif de Maryse Holder. Le malaise émane principalement de la nature paradoxale dont le film exhorte chez la spectatrice une attitude distanciée, mais perpétuellement compromise par les clins d'œil incessants que Maryse renvoie à une critique féministe de son propre comportement ${ }^{22}$.

Maryse Holder n'est pas un personnage banal. Professeure dans une université américaine où elle enseigne justement la critique littéraire féministe, Holder décide de prendre littéralement congé du féminisme pour se lancer dans une véritable fiesta sexuelle lors d'une croisade au Mexique. Les expériences sexuelles de Holder avec de jeunes Mexicains prennent alors les allures d'une vendetta contre elle-même: c'est-à-dire l'intellectuelle féministe. Son langage volontairement cru pour exprimer ses désirs, ses poses lascives et ses regards aguichants très «s.v.p. baisez-moi, je ne me peux plus» revêtent alors les atours d'une immense provocation au regard féministe. La provocation frôle même les limites de l'automutilation devant le corps «anorexié» et intoxiqué à l'alcool et aux drogues de Maryse. Car la force du film est là: celle de convier sans cesse le regard de la spectatrice 
à assister au phénomène de dégradation du corps de l'autre, le corps de l'autre évoquant ici la construction sociale de notre propre corps, celui des femmes.

Â ce chapitre, les expériences de Maryse revêtent les allures d'une véritable performance en direct, perfomance qui, on le sait, peut conduire dans certains cas à la mort. Et quand d'exaspération le regard de la spectatrice fuit Maryse, c'est elle-même qui nous interpelle, sollicite le regard encore une fois. Car le film ne se contente pas de suivre Maryse dans les diverses étapes d'un voyage. Il adopte une structure narrative qui interpelle directement les spectatrices à être les témoins en direct de sa quête amoureuse par l'explosion sexuelle. Qui plus est, Maryse, en adoptant le mode de l'auto-ironie et de l'humour sacarstique pour mieux relater ses (més)aventures, nous rappelle alors que si elle est sans cesse trompée et trahie par ses amants, elle, du moins, n'est pas dupe quant à l'absurdité de sa propre quête.

Basé sur un fait véridique, adapté de la correspondance de Maryse Holder $^{23}$, le film refuse tout suspense. On sait dès le départ que c'est la mort qui attend Maryse à l'extrême frontière de ses expériences. Tout ce qui nous reste à faire en tant que spectatrices, c'est d'écouter Maryse. Et elle nous parle, sans détour, avec l'arrogance et le cynisme de celle qui tout à la fois, parle, agit et regarde. Maryse désamorce toute critique féministe par sa propre autocritique: un discours hyperrationnel sur ses propres contradictions. Ainsi, si parfois le langage employé laisse croire à l'étalage de toute une série de clichés tant sur le machisme latino que le féminisme et le lesbianisme, l'emploi débridé des préjugés (et gestes) les plus vulgaires renforce l'idée de transgression des images par les mots, où la condamnation morale n'existe pas $^{24}$. En fait, pour Maryse, il s'agit de dire même ce qui ne peut être dit sous couvert d'un sens éthique qui serait le propre d'une intellectuelle conscientisée. Dès lors, le regard de la spectatrice ne peut être que continuellement désarçonné par l'ambiguïté délibérée du propos.

À ce chapitre, deux scènes que j'appellerais «siamoises» illustrent comment le film inscrit un regard féministe par l'exacerbation d'une représentation féminine. La première, au tout début du film, présente Maryse, avachie sur un lit de motel, complètement exaltée devant les multiples possibilités d'aventures qui l'atten- 
dent en terre mexicaine. Le ton est euphorique, arrogant, délirant. Aux objecteurs de conscience, le discours de Maryse oppose l'apologie des jeunes corps bruns et puissants, des cuisses fermes et pleines de promesses, des nuits de «baises» toutes plus excitantes et plus explicites les unes que les autres, tout cela agrémenté d'un tour du propriétaire digne à faire crever d'envie Narcisse luimême. Le recours à un langage politico-porno évite tout malentendu possible: si le discours triomphe, c'est d'une littérature explicite dont il sera question où la superposition des descriptions les plus osées vont de pair avec la parodie la plus noire.

Ce face à face avec la caméra, initié au tout début du film, où Maryse prend le regard de la spectatrice à témoin, revient dans la séquence finale. Alors que, pendant plus d'une heure, le discours de Maryse implacablement appelle une escalade de la provocation par la façon dont le corps de Maryse semble définitivement échappé au contrôle d'un discours encore critique, au moment où, dépitée, la spectatrice est sur le point de battre en retraite, survient cette incroyable scène où Maryse face à l'inévitable déploie devant nous un discours dont la froide lucidité raccroche le regard en même temps qu'il fait renaître un profond trouble. Dans cette séquence aux allures d'un requiem dénué de tout délire, c'est tout le procès et de son comportement et de la supercherie d'une sexualité bafouée qui l'emportent. Et c'est par un discours presque clinique sur l'affaissement de son propre corps que Maryse rend le mieux compte de l'immense duperie dans laquelle elle s'est vautrée tout ce temps. Ce corps auquel elle avait tenté désespérément d'épargner les marques du vieillissement, du corps chèrement adulé, il ne reste plus que les traces d'un voyage boulimique où l'amour somme toute n'a connu que les saveurs de la douleur.

Mais poussant l'absurde jusqu'au bout, Maryse ira, juste une dernière fois, «baiser» avec celui qui la tuera. Le regard critique de la spectatrice abandonne les charges arrogantes du propos pour dévier sur le corps tailladé de Maryse. C'est l'image du corps qui s'en va pour ne plus revenir qui imprègne le regard. Maryse, d'un dernier regard silencieux, concédera sans artifice qu'au-delà de la raison critique, c'est l'exacerbation subversive de sa propre sexualité qui aura le mieux désigné sa conception du désir. Ainsi 
encore une fois, Maryse laissera la spectatrice sans voix en s'appropriant le pouvoir de signifier elle-même, avec les termes justes, son propre rapport asservi au machisme. Les paroles initiales de Maryse retrouvent alors toute leur cohérence et toute leur lucidité dans cette diatribe où prône l'autoparodie: «I lose myself for a sense of female culture, female definition.»

\section{Épilogue: Thelma \& Louise ou The Ladies Vanish}

J'ai choisi de revoir Berlin Affair et $A$ Winter Tan, non pas pour les comparer, mais pour les mener de front, dans un corps à corps référentiel, pour interroger les possibilités d'un regard autre sur le cinéma, mais également pour pousser plus à fond les rappports entre cinéma et féminisme.

Les tentatives de Cavani et Burroughs doivent être comprises comme des stratégies qui interpellent directement chez la spectatrice cette tension entre le prescriptif et l'inscriptif. Ces films s'ébauchent comme des discours qui marquent, qui désignent le moment où à l'intérieur même d'un discours normatif s'ébauchent - parfois maladroitement - des espaces de subversion, des points de ruptures. Sans parler de stratégies sauvages (A. McRobbie), il s'agit ici de stratégies de références (Meaghan Morris) qui tout en «faisant avec» confrontent une représentation de la différence sexuelle qui conforte une façon de voir et de regarder les rapports hommes-femmes au cinéma.

C'est pourquoi j'ai cherché à insister sur des films qui évitent de construire une représentation qui «titille» le regard comme le ferait un vote électoral, sur des films qui rompent avec une identification traditionnelle pour un regard sexué. Ces films ouvrent sur des pratiques qui subvertissent les références traditionnelles aux rapports de sexes dans la société. Des films qui, en fait, ne construisent pas la femme comme autre dans l'éclatement des références, mais, de façon paradoxale et souvent ambiguë, dans l'exacerbation des traits typiques — sinon stéréotypés - à ce que serait «normalement» une représentation féminine.

Â ce chapitre, on est décidément très loin des tentatives superficielles et caduques des woman's films qui connaissent un regain de vie à Hollywood. J'ai en première partie évoqué très sommairement la distinction que je reprends ici de Lauretis entre 
woman's film et women's cinema. Si le women's cinema consiste à répondre à des pratiques cinématographiques qui veulent inscrire la femme comme sujet tant de la représentation qu'en tant que spectatrice, les woman's films consacrent, quant à eux, des tactiques qui, tout en faisant des femmes les héroïnes de leur film, ne contestent nullement l'inscription traditionnelle du mode de regard propre à une production cinématographique commerciale et dominante. J'aimerais illustrer brièvement cette distinction dans l'intention et dans le regard entre le projet du women's cinema et le type de woman's films effectif dans les années 90 à partir de l'exemple du film Thelma \& Louise (1991), réalisé par Ridley Scott.

Thelma \& Louise fut d'abord présenté comme un road movie féminin/féministe. En fait, une version 90 de l'époque des road movies soixante-huitards. Et c'est bien ce qu'il est: un roadmovie buddy buddy sans plus. Ce qui est fascinant dans Thelma \& Louise, c'est à quel point l'intrigue tient à une inversion sexuelle des personnages. Pour le regard de la spectatrice de même que pour le spectateur, cette inversion ne modifie en rien l'ordre et la position du regard. En faisant tenir à des femmes des rôles d'hommes, Scott ne bouleverse en rien le regard porté sur le film. Si l'identification chez la spectatrice peut jouer, c'est bien parce qu'elle ne conteste rien.

Tout dans ce film constitue une transposition fidèle de la façon dont un homme conçoit tantôt l'évasion, tantôt la répression, tantôt les redresseurs de tort. Peu importe que les délinquantes soient des femmes: les loisirs eux seront masculins: la pêche (sic!), le bar dans un trou perdu (l'aventure!), l'attrait pour la gâchette (si viril!). Qui plus est, la scène catalysatrice du film est pour moi un summum quant à la manière dont le film rassure un regard mâle sur une représentation au féminin de l'exode rural: je veux parler de la tentative de viol bien entendu. En effet, l'idée du viol est amenée ici d'un point de vue masculin et non féminin. Question: qu'est-ce qui apparaît comme le plus horrible pour un homme que le fait que sa femme soit violée? Formulons-le autrement: quelle est la chose la plus ignoble et la plus révélatrice sur le plan personnel dans l'atteinte à la propriété privée qui puisse survenir à un homme? 
Ce n'est pas un hasard si la victime d'une tentative de viol se révèle être Thelma, celle qui est mariée. Tout, en effet, l'y prédisposait: «bobonne» à la maison, n'ayant connu qu'un seul homme, brebis innocente face à la faune sauvage et cow-boy de l'Amérique profonde, Thelma était celle par qui le scandale arrive. La façon dont la scène est filmée (cadrages répétés sur les cuisses de Thelma) rappelle sans cesse que ceux qui regardent ne peuvent ni s'identifier à la victime ni adopter une distance critique face à la scène. Si le film permet à Louise d'assouvir une vengeance pour Thelma (et pour elle aussi) en tuant Allen, il ne faut pas croire pour autant au grand débarquement des amazones ${ }^{25}$. Car cette vengeance répond beaucoup plus à l'intention d'un homme face au violeur que de la femme face à un viol ${ }^{26}$. Car le film à ce chapitre est clair: ce n'est pas tant la scène qui est révoltante que le personnage (infect) d'Allen lui-même, présenté par Scott comme une bête de somme qui écume littéralement à la besogne (voir la tentative de viol). Et si les autres personnages masculins se retrouvent à maintes occasions dans des situations pour le moins absurdes et ridicules (l'archétype étant le mari de Thelma bardé de bijoux en or et au volant d'une corvette rouge), il faut voir le triomphe des bons sentiments «éducatifs» qui peu à peu chevauchent la fuite de Thelma et Louise quand elles décident de faire la leçon au camionneur-baiseur-dragueur-violeur potentiel. Les mots que disent alors Thelma et Louise pour condamner l'attitude machiste du camionneur s'inscrivent, encore une fois, dans une logique où ce n'est pas un rapport social qui est condamné mais bel et bien une attitude de vilain garçon. Quand Thelma et Louise lui demandent dans un ultimatum s'il aimerait que sa mère et sa soeur subissent le même sort de la part d'un camionneur frustré, on croirait presque entendre Richard Widmarck ou John Wayne prendre le parti de la veuve et de l'orphelin dans un duel à finir avec le méchant du coin dans un grand cru de l'épopée western. Bref, si Thelma \& Louise a quelque chose à voir avec l'inscription d'un regard féminin, on est bien plus près des weepies qui émeuvent tout en divertissant que du défi de dire la représentation autrement du women's cinema. Quant à la séquence finale, elle complète merveilleusement le tableau de chasse: un vol libre pour une séance de tir aux pigeons d'argile. 
Ainsi, Thelma \& Louise apparaît fort bien participer de l'esprit des woman's films des années 90: celui de situer dans un même mouvement des caractéristiques rendant compte des changements dans la position des femmes dans la société, tout en limitant ces mêmes changements de façon à exercer un contrôle sur les nouvelles expériences des femmes. Comme le dénote le nombre sans cesse croissant et hybride de woman's films qui croise les écrans ces dernières années, le genre lui-même, s'il appelle un nouveau traitement, $n$ 'en perpétue pas moins un regard traditionnel sur la représentation des femmes. Désormais sous couvert d'une réalité postféministe, des films comme The Blue Steel (Kathryn Bigelow, É.U., 1989), Working Girl (Mike Nichols, É.U., 1988), Postcards from the Edge (Mike Nichols, É.U., 1990), Fatal Attraction (Adrian Lyne, É.U., 1988), Steel Magnolias (Herbert Ross, É.U., 1989), Mortal Thoughts (Alan Rudolph, É.U.,1991), Switch (Blake Edward, É.U., 1991) s'inscrivent comme autant de versions noires d'un même regard mâle ethnocentriste ${ }^{27}$. Dès lors, tous ces films ne répondent qu'à des effets de surface où le regard ne fait que s'aplatir davantage devant la réussite d'une nouvelle chirurgie plastique ${ }^{28}$.

C'est pourquoi des œuvres comme Berlin Affair et $A$ Winter Tan doivent être considérées comme de réelles tentatives d'inscrire le regard de la femme dans une perspective critique. Si Berlin Affair pervertit et $A$ Winter Tan se moque, chacune de ces œuvres respecte ce que doit recouper un regard «autre» au cinéma: celui de pouvoir montrer ce qui est là caché bien à l'abri derrière les conventions du regard, celui de faire de la représentation de la femme non pas un point aveugle pour la spectatrice mais la ligne de fuite vers une reconnaissance du sujet.

Université de Montréal

\section{NOTES}

1 Cf. New Statesman and Society, vol. 3, n 127 (November 16, 1990).

2 Pour celles et ceux qui ne seraient pas familiers avec la politique intérieure britannique, je rappelle brièvement quelques éléments contextuels, ne serait- 
ce que pour mieux situer l'événement lui-même. Michael Heseltine, député conservateur et ex-ministre du gouvernement, est en fait celui qui a précipité la «chute» de Thatcher en novembre 1990. Celle-ci a dû démissionner et s'est retirée d'une lutte pour le leadership du parti conservateur à la suite d'abord de l'échec d'un premier vote de confiance suffisant de la part de ses députés, et, par la suite, devant la menace imminente d'un échec au deuxième tour. Heseltine, logique gagnant du putsch conservateur, s'est finalement retrouvé, après l'entrée en scène d'un autre candidat et actuel premier ministre John Major, bon deuxième.

3 Cf. Theresa de Lauretis, Technologies of Gender: Essays on Theory, Film and Fiction (MacMillan Press, 1987). Après bien des hésitations, j'ai préféré ici conserver la construction anglaise. Retenons simplement que pour de Lauretis, l'expression technology of gender renvoie directement à Foucault et sa théorie de la sexualité telle que développée dans son Histoire de la sexualité. Pour ce qui est de la traduction de gender, le sens du mot dépasse largement la simple référence au «genre» comme construction sociale de la différence biologique. En français, l'expression est chargée de la référence au pouvoir, donc au politique, dans les rapports entre les sexes, et, plus spécifiquement, du pouvoir d'un sexe (mâle) sur l' «autre» sexe (femelle). En somme, pour être le plus près possible de la dimension critique dont relève l'expression technology of gender, il faudrait traduire par technique de construction des rapports de sexes.

4 Cf. Christine Gledhill, «Developments in Feminist Film Criticism», in ReVision: Essays in Feminist Film Criticism, The American Film Institute Monograph Series (Frederick M. D.: University Publication of America and the American Film Institute, 1984)p. 18-48. Gledhill rappelle fort pertinemment que, «A crucial issue for feminist film criticism is the argument that "women as women" are not represented in the cinema, that they do not have a voice, that the female point of view is not heard.» (p. 18)

5 Cf. Teresa de Lauretis, «Guerilla in the Midst: Women's in the 80's», in Screen, vol. 31, n 1 (1990) p. 6-25.

6 Cf. Meaghan Morris, Camera Obscura, «La Spectatrix», nº 20-21 (mayseptember 1989) p. 242.

7 Cf. Gayatri Chakravorty Spivak, «Can the Subaltern Speak? , in Nelson, Cary and Lawrence Grossberg (eds.), Marxism and the Interpretation of Culture (Urbana: University of Illinois Press, 1988).

8 Cf. Meaghan Morris, The Pirate's Fiancée: Feminism, Reading, Postmodernism (London \& New York: Verso, 1988).

9 Statégies d'énonciation que Morris institue comme autant de points of address 
qui structurent le processus du regard au cinéma pour la spectatrice. Cf. Camera Obscura, «LaSpectatrix», $\mathrm{n}^{\circ} 20-21$ (may-september 1989) p. 243. En somme, comme l'explique Morris, il n'existe pas de female spectator en soi, c'est-à-dire par essence. Sa critique en fait vise plutôt à reconnaître une spectatrice construite par un ensemble complexe de stratégies de références qui interpellent le regard comme celui de la spectatrice. Ainsi, comme l'explique Morris, «(...) the term "female spectator" has been most productive not as a mediation between a concept of the empirical spectator and one of a hypothetical point of address, but rather as a metonym of a zone (and phase) of analysis produced by contemporary theory, in which the relations between empirical and rhetorical (or historical and theoritical) feminist research in cinema can be conceptualized - along with a politics of the purpose (and "points of address") of such research in the present» (p. 243).

10 L'expression évoque bien sûr le titre d'un texte d'Adrienne Rich (1981), «La Contrainte à l'hétérosexualité et l'existence lesbienne», Nouvelles Questions féministes, vol. $1, \mathrm{n}^{\circ} 1$ (1981).

11 Cf. Annette Kuhn (ed.) avec la collaboration de Susannah Radsone, Women in Film: An International Guide (New York: Fawcett Columbine, 1990) p. 68.

12 Cf. Kaja Silverman, The Accoustic Mirror: The Female Voice in Psychoanalysis and Cinema (Bloomington \& Indianapolis: Indiana University Press, 1988)p. 218-234. Silverman souligne également que cette identification opère avant tout chez la réalisatrice qui parlerait toujours de ses films en adoptant le point de vue de ses protagonistes masculins.

13 Silverman remarque fort judicieusement chez Cavani que «it is a striking fact that the male characters who dominate Cavani's films retreat from power that accede to it; that they entertain a highly problematic relation to discourse; and that they interact with women in ways which defy the usual heterosexual conventions» (Silverman, p. 220).

14 Ainsi, «Cavani's cinema would consequently seem to be fueled by the dream of androgyny» (Silverman, p. 224).

15 L'expression est celle que Louise emploie pour traduire sa curiosité à l'endroit de Mitsuko. L'allusion est intéressante dans la mesure où elle décrit parfaitement la référence stéréotypée à la geisha japonaise.

16 Rappelons en effet la célèbre interprétation que donne Bellour du «rôle» de la spectatrice au cinéma, et du coup du plaisir au féminin... Commentant notamment la position de la spectatrice face aux films de Hitchcock, Bellour avance le plus sérieusement du monde: «I think that a woman can love, accept and give a positive value to these films only from her own masochism, and from a certain sadism that she can exercise in return on the masculine subject, within 
a system loaded with traps»; cité et traduit du français par Constance Penley, in The Future of An Illusion: Film, Feminism, and Psychoanalysis (Minneapolis: University of Minnesota Press, 1989) p. 43.

17 Cf. Teresa de Lauretis, «Cavani's Night Porter : A Woman's Film?», in Film Quaterly, vol. XXX, n² 2 (1976-77) p. 35-38.

18 Le texte de Teresa de Lauretis soulève des questions pour le moins pertinentes dans la mesure où justement l'auteure s'interroge sur la propension réelle chez Cavani à adopter le point de vue de ses personnages masculins. À cet égard, l'interprétation de Silverman trouve d'autant plus prise pour une critique féministe de la représentation des femmes dans le cinéma de Cavani.

19 Sue Stewart, «Is Lilianna Cavani Really a Woman?», in Films Forbidden, «The Filmmaker and Human Rights in Aid and Amnesty International» (Toronto, October 1984) p. 18-28.

20 Le traitement de la sexualité chez Cavani n'est pas loin d'une vision existentialiste où finalement l'être-en-soi domine sur les conditions extérieures qui définissent la différence sexuelle. Dès lors, comme le soutient Sue Stewart «she [Cavani] articulates the ideas that power can shift and become redistributed on many different levels and that simplistic notions of passivity and dominance can distort our perception of sexual realities» (Stewart, p. 37).

21 Cf. Elspeth Probyn, «New Traditionalism and post-feminism; TV does the Home», in Screen, vol. 31, n² 2 (1990) p. 147.

22 Ibid., p. 147.

23 Cf. Give Sorrow Words: Maryse Holder's Letters from Mexico (New York: Grove Press, 1979).

24 Cf. Shonagh Adelman, «Maryse Holder Liked to Fuck», in Fuse, n 51 (1988) p. 34-36. Adelman établit clairement que ce qui est mis de l'avant dans le film c'est l'expression politically incorrect (p.34) de Maryse. Ainsi, observe-t-elle, «The use of porn humour goes beyond transgression in its racist stereotyping of Mexican men. As in her view of feminists and lesbians, her satire is doubleedged. As a sexual outlaw in "third world" country, the issues are compounded by the fact that though she is oppressed as a woman, she is privileged as an American. These issues are not obscured or condoned by the film, but deliberately exposed» (p. 36).

25 Quoiqu'à ce chapitre, la tentation soit grande d'y faire allusion, comme en témoigne Kathleen Murphy (in Film Comment, January-February 1992, p. 42) qui, appelée à dresser la liste des 10 meilleurs films de l'année a placé en même 
position (la $8^{\circ}$ ) toute un série de woman's films si joliment rebaptisés pour les circonstances warriors women.

26 Comme l'a rappelé si crûment Anne Claire Poirier dans Mourir à tue-tête (1979), la première chose qui vient en tête aux hommes quand survient un viol, c'est d'abord de régler le compte du violeur.

27 Cf. Amelia Jones, «"She Was Bad News": Male Paranoia and the Contemporary New Woman", in Camera Obscura, n 25-26 (1991) p. $297-$ 320. Dans cet article, Jones introduit la notion de new woman's films pour marquer cette série assez impressionnante de films hollywoodiens où les femmes sexuellement et professionnellement actives apparaissent comme de véritables anges de la mort (the bad news) pour l'ordre patriarcal. Jones souligne alors que «The new woman's films are structured by what Alice Jardine has called «male paranoia» - the fearful response of patriarchy to the loss of boundaries endemic to the condition of subjectivity in contemporary socalled postmodern, American life» (p. 297).

28 Avec Switch, la métaphore devient d'autant plus pénétrante que là on assiste réellement à une métamorphose sexuelle. Assassiné par un triumvirat d'exmaîtresses aux idées vengeresses, un homme dont l'attitude on ne peut plus cavalière et infecte à l'égard des femmes est renvoyé illico presto sur terre dans l'enveloppe charnelle de l'objet de ses désirs. Laffreux misogyne macho expérimente en direct les sept douleurs du corps d'une femme. Toutefois, si l'idée au début laissait présager certaines trouvailles (notamment celle où le/ la héros/héroïne se trouve confronté/e à la réalité lesbienne), la finale du film célèbre l'apologie de l'enfantement comme rédemption des péchés en ce bas monde.

\section{OUVRAGES CITÉS}

Le Dœuf, Michèle. L'Étude et le rouet, tome 1. Paris: Seuil, 1989.

Morris, Meaghan. «La Spectatrix». Camera Obscura, nos 20-21 (mayseptember 1989).

Stewart, Sue. «Is Lilianna Cavani Really a woman?». Films Forbidden «The Filmmaker and Human Rights in Aid and Amnesty International» (Toronto, October 18-28, 1984). 